\title{
EVOLUCIÓN HUMANA Y ANTROPOLOGÍA VIRTUAL: UNA PROPUESTA PARA LA DOCENCIA Y LA INVESTIGACIÓN
}

\author{
Alexia Serrano Ramos 1,3; Jiménez Arenas, J.M. ${ }^{1,2}$ y Esquivel Guerrero, J.A. 1,2,3
}

\section{Resumen:}

Partiendo de la premisa de dinamizar ciertos recursos existentes en el Departamento de Prehistoria y Arqueología (la colección de réplicas osteológicas - cráneos - y un escáner 3D de superficie) nos propusimos como objetivo componer una colección virtual de referencia de materiales osteológicos accesible, comprensible y funcional, para enriquecer la formación teórico-práctica del alumnado, y contribuir a la mejora de la enseñanza de la evolución humana en los diferentes grados y posgrados de la Universidad de Granada, pero también válido para la investigación

Para ello, se procedió a digitalizar, con un escáner 3D de luz estructurada Artec2000, 25 réplicas de cráneos de individuos significativos para el estudio de la evolución humana y pertenecientes a los principales taxones extintos (australopitecinos -en sentido amplio- y Homo). Además, se realizaron fichas de cada uno de ellos con información relativa a su descubrimiento y adscripción, así como de algunas de las principales variables métricas craneales (volumen endocraneal, tres del neurocráneo y otras del tres viscerocráneo) que se hallaban dispersas en la literatura científica. Dicha información junto con las reproducciones digitales configuran una base de datos alojada en una página web "la craneoteca del Dpto. de Prehistoria y Arqueología de la UGR", de libre acceso desde cualquier computadora con Internet (www.prehistoriayarqueología.es/craneoteca).

\section{Palabras clave:}

Cráneos, docencia, escáner 3D, evolución humana, nuevas tecnologías.

\section{Abstract:}

Based on the premise of boosting certain resources of the Department of Prehistory and Archaeology (the collection of osteological replicas - skulls - regarding Human Evolution and the 3D scan) we focused our aim in creating a virtual reference collection of the osteological material in an accessible, comprehensible, functional way, in order to improve the theoretical-practical training of students and therefore contribute to enrich teaching of Human Evolution in the different graduate and postgraduate studies at the University of Granada, but also suitable for Research.

1 Departamento de Prehistoria y Arqueología, UGR - alisera@correo.ugr.es

2 Instituto de la Paz y los Conflictos, UGR - jumajia@ugr.es

3 Laboratorio 3D de Modelización Arqueológica, UGR - esquivel@ugr.es 
We proceeded to digitize, with Artec 2000, a 3d structured light scan, 25 skull replicas of significant individuals in the study of Human Evolution, belonging to the main extinct taxa (australopiths and Homo). In addition, we made a database with information concerning its discovering and taxonomic adscription, as well as some of the main metric variables concerning the cranium (endocraneal volume, neurocranium and viscerocranium) that were dispersed in the scientific literature. All the information and the digital reproductions configure a database hosted in a web site "La craneoteca del Dpto. de Prehistoria y Arqueología de la UGR", free access from any computer with internet (www.prehistoriayarqueología.es/ craneoteca).

\section{Key words:}

3D scan, human evolution, new technologies, skulls, teaching.

\section{Introducción}

El siglo XXI es sin duda el momento clave en cuanto a grandes avances tecnológicos y del desarrollo de internet y del mundo virtual. Numerosas aplicaciones tecnológicas del ámbito sanitario (TAC's), militar (drones), del ámbito recreativo (videojuegos, cine, realidad aumentada) o de las aplicaciones industriales (escáneres y máquinas de prototipado rápido) han comenzado a ser aplicadas al mundo de la Arqueología, con distintos objetivos y resultados.

Se puede definir la arqueología virtual como la disciplina científica que tiene por objeto la investigación y el desarrollo de formas de aplicación de la visualización asistida por ordenador a la gestión integral del patrimonio arqueológico. Las nuevas tecnologías pueden ser una herramienta muy útil precisamente por su versatilidad en la metodología arqueológica. Por un lado permiten la documentación completa y la integración del material arqueológico, como en el caso del escaneo de niveles arqueológicos y los artefactos asociados a ellos que se llevó a cabo en diversos yacimientos del Paleolítico Medio en Francia (McPherron et al., 2009) o en proyectos que utilizan las tecnologías digitales para obtener documentación más detallada (Doneus et al., 2003; Martínez Lerones et al., 2009; Mostaza et al., 2010). También resultan muy útiles a la hora de realizar la sistematización de los análisis métricos e incluso minimizar los errores de medición (Morales et al., 2013; Grosman et al., 2008). Por otro lado, los modelos digitales son ampliamente utilizados en reconstrucciones y musealizaciones virtuales (Böhler et al., 2008), ligados al desarrollo de la realidad virtual (VR) y que permite la interactuación entre el público, los materiales arqueológicos y el escenario contextual (Barceló et al. 2000; Lepouras \& Vassilakis, 2005; Bruno et al., 2010). En este sentido las impresoras en 3D o de prototipado rápido resultan un valor añadido, permitien- 
do la obtención transitar de la realidad virtual a la virtualidad real mediante la creación de réplicas. Asimismo, los modelos digitales pueden resultar muy útiles para realizar propuestas de restauración virtual, e incluso para monitorizar dicho proceso en elementos complejos, como en la conservación de corchos y otros materiales arqueológicos procedentes de yacimientos anegados (Stéphanie et al., 2013). Incluso pueden facilitar la monitorización para la conservación general del patrimonio cultural, realizando escaneos periódicos que permitan estudiar las alteraciones que pueden poner en peligro su conservación, como en el caso extremo del teatro romano de Pinara, en el suroeste de Turquía, situado en una zona de peligro sísmico (Hinzen et al., 2013).

Más específicamente, en el ámbito de la antropología, la paleontología y otros campos de estudios afines, los métodos $3 D$ imaging están permitiendo a los científicos mediante la adquisición de imágenes mediante tomografías computerizadas (TC), resonancias magnéticas (RM) e imágenes terahertzianas ( $\mathrm{THz}$ ) expandir e implementar sus investigaciones (Tocheri, 2009): (Allam et al., 2011; Buisktra, 2010; Conlogue et al., 2008; Faccia \& Williams, 2008; Öhrström et al., 2010; Panagiotopoulou, 2009; Saita et al., 2011). Estas tecnologías presentan la desventaja de no ser portables (imposibilitando así el estudio de aquellos materiales que no se pueden transportar) y que el procesado y estudio de este tipo de imágenes $3 \mathrm{D}$ requiere de un software caro y de personal especializado. Con la generalización de los escáneres (especialmente en cuanto al precio de los dispositivos), la digitalización de homininos fósiles y actuales y materiales osteológicos faunísticos ha sido ampliamente utilizado (Dawson and Levy, 2005; Hennersy and Stringer, 2002; Kappelmann et al., 2000, 2001; Larson et al., 2009; Lyons et al., 2000; Motani, 2005; Pfisterer et al., 2007, 2008; Plyusnin et al., 2008; Smith and Strait, 2008; Strait and Smith, 2009). En el ámbito de la investigación los modelos digitales están generalizándose en los análisis de elementos finitos, la anatomía comparada, la morfometría geométrica y las técnicas elípticas de Fourier (Friess, 2010; Garvin \& Ruff, 2012; Hennesey et al., 2002; Relvas et al., 2011; Sholts et al., 2011; Zollikofer \& Ponce de León., 2002). Como alternativa de almacenamiento, catalogación y preservación, el registro digital de material óseo puede suponer una implementación en la conservación de estos frágiles restos en Museos y otras instituciones (Kuzminsky \& Gardiner, 2012). A nivel de difusión, es obligatorio mencionar a The Smithsonian Institution's Human Evolution Center, en Washington D.C., donde se ha creado una extensa base de datos online de fósiles de primates y homininos que está disponible para su visualización online y de manera gratuita. La posibilidad de impresión en 3D del material osteológico digital puede ser beneficiosa no sólo con objeto educacional o divulgador, sino también en la investigación. Tal es el caso del Max Planck Institute of Evolutionary Anthropology, donde han impreso una réplica de un diente humano en un tamaño veinte veces más grande que el original para facilitar el estudio de la morfología de las cúspides (http://www.eva.mpg.de/evolution). Una nueva vuelta de 
tuerca vino de la mano de la ampliación del concepto de reconstrucción virtual. Se trataba de solventar un problema clásico en Paleontología y, por extensión, en Paleoantropología: la deformación, la fragmentación y los posteriores intentos de restauración y reconstrucción. Para ello, Christoph Zollikofer y Marcia Ponce de León, desde la Universidad de Zurich, comienzan a desarrollar un paquete informático interactivo que permite no solo la visualización y la segmentación, sino también la restauración virtual de los materiales (Zollikofer et al. 1995). Esto resulta primordial, si luego se pretende también aplicar un análisis para evaluar estadísticamente la variabilidad formal de los objetos. Así, para la cuantificación y caracterización topológica y matemática de los fósiles es fundamental que éstos no estén distorsionados y que cada una de las partes esté situada en el lugar correcto (Figura 1). De otra manera, debido a la sensibilidad a las variaciones formales de las herramientas usadas en morfometría geométrica, los análisis cuantitativos se verían seriamente comprometidos; también, las descripciones y comparaciones que se hicieran de los fósiles.

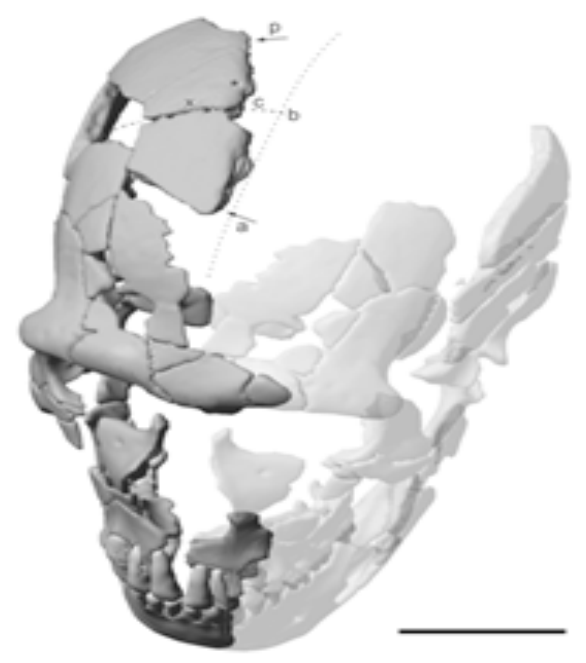

Fig. 1. Reconstrucción virtual del cráneo neandertal Saint Cesaire 1. a y p: anteroposteriores límites de la herida; c: sutura coronal; b: bregma (Zollikofer et al. 2002).

Los resultados del equipo zuriqués han tenido una enorme repercusión científica y mediática, no solo para el caso de los neandertales (Zollikofer \& Ponce de León 2002; 2008; Zollikofer et al. 2002), sino también porque han participado en algunos de los debates más interesantes de la Paleoantropología: el estatus taxonómico y forma de locomoción del primer hominino fósil conocido, Sahelanthropus tchadensis (Toros Menalla, Chad) (Zollikofer et al. 2005) y la variabilidad de la población de Homo tempranos de Dmanisi (Georgia) (Lordkipanidze et al. 2013). 
El uso exponencial de modelos digitales en la investigación relacionada con la Evolución Humana en parte se explica por el delicado estado de conservación de gran parte del registro fósil perteneciente a tiempos tan remotos. Esto se suma a lo disperso de las evidencias paleoantropológicas a lo largo de todo el globo, lo cual dificulta y restringe el acceso al material original a gran parte de los investigadores, y por descontado al alumnado y público en general.

Aunque en general, las colecciones de referencia son una herramienta fundamental en las ciencias naturales y sociales, pero especialmente en la arqueología, donde la identificación de artefactos, restos de fauna, pólenes y otros materiales es un componente base de análisis (Banning, 2000). Lo restringido del registro fósil humano ha favorecido la creación y disposición de colecciones de referencia para el estudio y difusión de los estudios sobre Evolución Humana. La realización de réplicas de los cráneos fósiles siempre ha formado parte por ello del proceso de estudio paleoantropológico. En ocasiones, como el caso de los cráneos de "el hombre de Pekin", tras desaparecer los cráneos originales hacia 1941 en el contexto de la ocupación japonesa de China, sólo contamos con las réplicas que realizaron diversos investigadores (en nuestra colección contamos con la realizada por F. Weidenreich). Además, en cualquier museo del mundo es más que probable encontrar, en la sala de Prehistoria, la clásica vitrina que alberga las réplicas de los cráneos más explicativos del proceso de hominización, como es en el caso de la Sala II del Museu de Prehistòria de València o en el recientemente inaugurado David H. Koch Hall of Human Origins en el Smithsonian's National Museum of Natural History en Washington D.C. Asimismo, muchos centros de formación y/o investigación, como las universidades, suelen atesorar a lo largo de tiempo, colecciones de referencia que se pueden considerar fundamentales como materiales de apoyo tanto a la docencia como a la investigación. En el caso que nos ocupa, el Departamento de Prehistoria y Arqueología de la Universidad de Granada dispone de una colección de materiales, réplicas paleoantropológicas de gran calidad que se ha ido configurando a lo largo de la historia de esta institución.

Ejemplos como éste resultan fundamentales para la investigación de distintos aspectos biológicos y culturales durante la evolución de los homininos, pero también se convierten en una herramienta altamente útil para la docencia en el ámbito de la Evolución humana, como por ejemplo en la capacitación para reconocer características anatomo-mofológicas significativas y distinguir la variabilidad. Llegados a este punto es necesario relacionar la estructural carencia de prácticas relacionadas con la Antropología física y la Evolución humana con las que cuentan las asignaturas de Arqueología o los nuevos planes de grado, al menos en nuestra universidad, aunque presumimos que extensibles a otros centros educativos, y de las que tanto se quejan los alumnos, sobre todo teniendo en cuenta el auge que los estudios sobre evolución humana están teniendo en los últimos años. 
De momento estos materiales, guardados en el Departamento, tan sólo han sido utilizados como recurso de manera puntual en las prácticas de "Prehistoria I" y en la asignatura "Las comunidades de cazadores y recolectores", quedando a nuestro juicio, muy desaprovechado el potencial didáctico de la colección.

A partir de lo dicho anteriormente, mostraremos el desarrollo y resultados del proyecto "Evolución Humana y Antropología Virtual" desarrollado y subvencionado en el marco de "Proyectos de Innovación y Buenas Prácticas Docentes" (2012) de la Universidad de Granada que se propone contribuir a la mejora de la enseñanza de la Evolución humana en los diferentes grados y posgrados de la Universidad de Granada. Asimismo, esperamos fomentar la iniciación a la investigación.

Específicamente el objetivo ha sido componer una colección virtual de referencia de materiales osteológicos accesible, comprensible y funcional, para implementar la formación teórico-práctica del alumnado, pero también válido para la investigación. Una herramienta que sea útil para la consulta formativa o de investigación debe ser potente y versátil, con protocolos claros de información y descripción, los cuales, en consecuencia, permitan una rápida respuesta ante cualquier cuestión planteada por los usuarios potenciales de la misma.

De igual forma, resultó necesario organizar la colección mediante un sistema de clasificación que permita un fácil acceso a cada ítem y a toda la información alfanumérica asociada al mismo. Por ello se replanteó la estructura de la colección desde dos perspectivas: primero, a partir del material existente y decidir qué tipo de objetivos formativos e investigadores se pueden conseguir del mismo; y segundo, plantear los protocolos de implementación del material, de modo que se adecuen a las necesidades y objetivos de dicha colección hasta convertirla en un referente completo amoldado a los fines perseguidos con la constitución de la misma.

Consideramos que la creación de un recurso como este supone un potente instrumento didáctico en el ámbito de la evolución humana que permite renovar y mejorar la metodología docente en prácticas en diversos grados universitarios (Historia, Biología, Geología, Antropología Social y Cultural e Ingeniería Informática), consiguiendo integrar de forma eficiente y efectiva la teoría y la práctica. Contamos con incrementar notablemente la capacidad de aprendizaje, ya que se convierte en una aprehensión directa de la información, siendo el propio alumnado el que recoge y visualiza la información que precisa para comprender los distintos aspectos anatómicos, morfológicos y morfométricos de cada uno de los huesos o complejos óseos concentrados en una sola actividad didáctica. Asimismo, esta clase tipo de herramientas resulta muy atractiva para el alumnado actual puesto que se trata de un tipo de soporte muy familiar para las nuevas generaciones. 


\section{Materiales: Colección de réplicas del Departamento}

La colección de réplicas osteológicas del Departamento de Prehistoria y Arqueología de la Universidad de Granada se ha ido configurando a lo largo del tiempo a través de donaciones y adquisiciones. Principalmente está compuesta por la colección donada por Fernando Aparicio, y la otra gran remesa es fruto de adquisiciones del Departamento a través de diversos programas propios de la UGR y también de la Unión Europea, en una tienda especializada en réplicas osteológicas de gran calidad. La colección incluye ejemplares de primates, Australopithecus, Kenyanthropus, Paranthropus y Homo. Principalmente son réplicas craneales: cráneos (completos y calvarias) y mandíbulas (en ocasiones asociadas al mismo individuo aunque estén separadas). Existen algunas réplicas de parte del esqueleto postcraneal de algunos individuos destacando el KNM-WT 15000.

El primer paso del proyecto consistió en inventariar la colección y seleccionar aquellos especímenes que podían ser incluidos en el proceso de digitalización. Algunos ejemplares estaban repetidos al combinar las dos pequeñas colecciones. En estos casos se utilizaron los ejemplares de las adquisiciones más recientes. La Tabla 1 recoge la relación de individuos digitalizados.

\begin{tabular}{|l|l|l|l|l|}
\hline Especie & Especimen & P. osteológica & Yacimiento & Colección \\
\hline Kenyanthropus playtops & KNM-WT 40000 & cráneo & Lago Turkana, Kenia & 2 \\
\hline $\begin{array}{l}\text { Australopithecus } \\
\text { afarensis }\end{array}$ & Reconstr. T. White & cráneo & [Hadar, Etiopía] & 1 \\
\hline $\begin{array}{l}\text { Australopithecus } \\
\text { africanus }\end{array}$ & Taung 1 & $\begin{array}{l}\text { frontal y } \\
\text { maxilar }\end{array}$ & Taung, Sudáfrica & 1 \\
\hline $\begin{array}{l}\text { Australopithecus } \\
\text { africanus }\end{array}$ & Sts 5 & cráneo & $\begin{array}{l}\text { Sterkfontein, } \\
\text { Sudáfrica }\end{array}$ & 1 \\
\hline $\begin{array}{l}\text { Paranthropues } \\
\text { aethiopicus }\end{array}$ & KNM-WT 17000 & cráneo & Lago Turkana, Kenia & 1 \\
\hline Paranthropus robustus & SK 48 & cráneo & $\begin{array}{l}\text { Swartkrans, } \\
\text { Sudáfrica }\end{array}$ & 1 \\
\hline Paranthropus bosei & KNM-ER 406 & cráneo & Koobi Fora, Kenia & 2 \\
\hline Homo habilis & KNM-ER 1813 & cráneo & Koobi Fora, Kenia & 1 \\
\hline Homo habilis & OH-24 & cráneo & Olduvai, Tanzania & 2 \\
\hline Homo ergaster & KNM-WT 150000 & cráneo & Lago Turkana, Kenia & 1 \\
\hline Homo ergaster & KNM-ER 3733 & cráneo & Koobi Fora, Kenia & 1 \\
\hline Homo erectus & Trinil 2 & calvaria & Trinil, Java & 2 \\
\hline
\end{tabular}




\begin{tabular}{|l|l|l|l|l|}
\hline Especie & Especimen & P. osteológica & Yacimiento & Colección \\
\hline Homo erectus & Sangiran 17 & $\begin{array}{l}\text { Calvaria }+ \\
\text { maxilar }\end{array}$ & Sangiran, Java & 2 \\
\hline Homo erectus & OH-9 & calvaria & Olduvai, Tanzania & 2 \\
\hline Homo erectus & Reconstr. Weidenreich & cráneo & $\begin{array}{l}\text { Zhoukoudian C.I. } \\
\text { China }\end{array}$ & 1 \\
\hline Homo georgicus & D2700 & cráneo & Dmanisi, Georgia & 2 \\
\hline Homo heidelbergensis & Steinheim 1 & cráneo & $\begin{array}{l}\text { Wurttenberg, } \\
\text { Alemania }\end{array}$ & 2 \\
\hline Homo heidelbergensis & Craneo no 5 SH & cráneo & Atapuerca, España & 2 \\
\hline Homo floresiensis & LB1 & cráneo & $\begin{array}{l}\text { Isla de Flores, } \\
\text { Indonesia }\end{array}$ & 2 \\
\hline Homo neanderthalensis & Teshik Tash & cráneo & $\begin{array}{l}\text { Bajsun-Tau, } \\
\text { Uzbekistán }\end{array}$ & 2 \\
\hline Homo neanderthalensis & La Chapelle-Aux- & cráneo & Corréze, Francia & 2 \\
\hline Homo neanderthalensis & Quina H18 & cráneo & Charante, Francia & 2 \\
\hline Homo sapiens & Skhul V & cráneo & $\begin{array}{l}\text { MonteCarmelo, } \\
\text { Palestina }\end{array}$ & 1 \\
\hline Homo sapiens & Cro-magnon 1 & cráneo & Dordogne, Francia & 1 \\
\hline
\end{tabular}

Tabla 1. Ejemplares de la colección utilizados en la Craneoteca; adscripción taxonómica según el inventario del Depto. de Prehistoria y Arqueología; la identificación del ejemplar; parte esquelética digitalizada; yacimiento y país de procedencia; y colección del Depto. a la que corresponde (el $n^{\circ} 1$ es para la colección más antigua - denominada Fernando Aparicio - y el $n^{\circ} 2$ son las nuevas adquisiciones de "Paleomundo").

Los ejemplares son especímenes muy ilustrativos del proceso de evolución humana. Se utilizan puntualmente como recurso complementario en las prácticas de algunas asignaturas de Prehistoria en diversos grados de la UGR. Pero la mayor parte del tiempo, la colección de réplicas osteológicas se encuentra almacenada en un despacho del departamento.

\section{Métodos: Escáneres 3D. El proceso de digitalización}

Para crear la colección virtual hemos empleado un escáner de luz estructurada 3D de low range, el Artec 2000. Esta herramienta permite capturar la emisión de algún tipo de luz o radiación hacia el objeto para así detectar la reflexión que 
genera (escáneres activos). Es decir, implica la medición de puntos de un objeto en un sistema de coordenadas en 3D adquiriendo la forma y características de un objeto mediante la proyección de un patrón de luz y su registro en un sistema de adquisición. Además, el escáner incorpora una cámara digital, con una resolución de 1280x800 píxeles. Esto permite la obtención de imágenes en color que permiten generar posteriormente la textura real del elemento escaneado.

La velocidad de captura oscila entre 7 y 15 fotogramas por segundo, trabajando de manera óptima en un rango de distancia comprendido entre 0.40 y 2 metros y con un error máximo de 0.5 milímetros. Este tipo de escáner sin contacto emplea señales lumínicas que se propagan en el medio, implicando una menor distorsión de la pieza. Como desventaja, resulta dificultoso el escaneo de elementos cuya superficie sea de color negro, ciertos materiales transparentes o con demasiado brillo.

Artec Studio es el software de referencia del escáner con el que se ha realizado el procesamiento de los datos tridimensionales, y que dispone de forma integrada de los algoritmos, comandos y operaciones necesarias para el registro, tratamiento y almacenamiento de los datos y resultados.

El proceso de digitalización con ARTEC 2000 consta de diferentes fases aunque presenta un alto grado de automatización (Figura 2). Tras unos meses de pruebas establecimos un protocolo funcional, eficiente y que optimizaba el proceso y nos permitía obtener resultados homogéneos. En nuestro caso decidimos colocar el elemento a escanear en una plataforma estática y mover el escáner alrededor, controlando la distancia escáner - objeto, la inclinación y la velocidad del movimiento para realizar un escaneado más eficiente y eficaz.

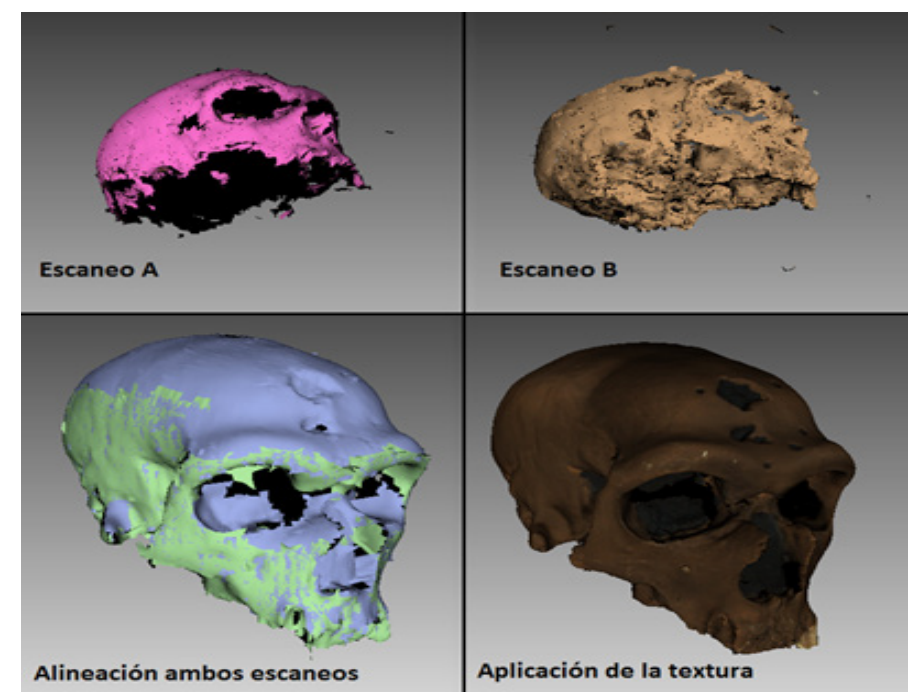

Fig. 2. Se muestra los pasos más importantes del proceso de digitalización: los diferentes planos, la alineación y la texturización del modelo. 
El siguiente paso consiste en la adquisición de los datos. Se escanean los diferentes planos del elemento. Por lo general hemos realizado dos, un plano superior y uno inferior (colocando el cráneo sobre una estructura ligeramente convexa para que se sostuviese). Es necesario obtener zonas que se solapen para luego poder unirlas, aunque el algoritmo de registrado es bastante flexible y tolerante a fallos. Reducir el número de planos al máximo nos permitía poder trabajar con ficheros ligeros que no daban problemas de procesado con nuestro sistema operativo.

Los ficheros resultantes están formados por una nube de puntos, que suelen incluir información referente al lugar de escaneo, por lo que hay que realizar una limpieza del ruido para eliminar todos aquellos puntos que no tienen relación con el elemento escaneado.

Posteriormente es necesario llevar a cabo el registro de los datos. Para ello se realiza la alineación de los distintos escáneres a partir de puntos comunes en un único sistema de coordenadas. Después, el algoritmo global optimization of frame positions selecciona un conjunto de puntos geométricamente únicos de cada marco y, comparándolos entre ellos, obtiene información relativa de cada par de marcos. Entonces optimiza la posición de todos los marcos en un sistema de coordenadas global, corrigiendo errores y fallos del alineamiento.

Los escaneos originados deben integrarse en un modelo geométrico único mediante un algoritmo que interpola entre múltiples vistas formando un único modelo geométrico 3D en base a triángulos en una denominada "concha". Se puede configurar la resolución/tamaño de la triangulación de la cuadrícula en milímetros. Una vez completada la fusión es conveniente comprobar posibles defectos del modelo 3D para eliminarlos: filtro de outliers, rellenado de pequeños agujeros y alisado de superficies.

Finalmente, el modelo digital está listo para ser exportado. Se puede hacer en diversos formatos, como .PLY (archivo de polígonos, en alta resolución se puede utilizar con fines de archivo y de investigación), .OBJ (objeto 3D, ampliamente utilizado para la visualización tridimensional), .STL (que es el formato utilizado para la impresión 3D), y que generalmente pueden ser legibles/utilizados en diferentes software y dispositivos online. En nuestro caso, exportamos los modelos digitales en .PLY para su almacenamiento, y para la visualización de la colección utilizamos los formatos .OBJ con las texturas asociadas.MTL y .PNG.

La principal dificultad del proceso de digitalización viene determinada tanto por la topografía de los cráneos (concavidades y convexidades profundas que dificultan el proceso de escaneado), lo que en ocasiones ha imposibilitado la obtención de un modelo en 3D de calidad (como en el caso del ejemplar de Sahelanthropus tchadensis), o algunos colores (especialmente las zonas negras) y brillos, que han provocado pequeños fallos en la generación de las texturas. 
Una de las ventajas al digitalizar los cráneos ha sido que, la forma y orientación de este complejo óseo es de fácil reconocimiento, de forma que se pueden trabajar con la nube de puntos sin sobrecargar demasiado los archivos. Por lo demás, tanto el manejo del escáner como del software son muy intuitivos y de rápido aprendizaje, de manera que logramos optimizar el proceso, logrando que en menos de 30 minutos pudiésemos obtener los datos, procesarlos y generar el modelo 3D texturizado.

\section{Resultados: la craneoteca del Dpto. Prehistoria y Arqueología (UGR) en la web}

El proyecto contemplaba la generación de una base de datos que recogiera la información disponible sobre cada individuo fósil y que ayudase a contextualizar los hallazgos. En primer lugar se realizaron fichas de cada uno de los individuos escaneados con información general relativa a su descubrimiento: nombre del ejemplar (y nombre popular si lo tiene), adscripción taxonómica, lugar del hallazgo, quién lo descubrió, quién lo presentó y la datación. También se recolectaron las principales variables métricas craneales, dispersas en la literatura científica: el volumen endocraneal, tres medidas del neurocráneo (longitud, altura y anchura máxima) y tres relativas al esplacnocráneo (longitud facial, altura facial superior y anchura bicigomática). En todos los casos se indica la fuente de la que hemos sacado la información, que también está recogida en una pestaña a parte [bibliografía] en la página del proyecto. Además, se incluye en cada ficha la geolocalización del hallazgo (a través de mapas), siendo posible utilizar esta información como una variable para cuestiones ecogeográficas.

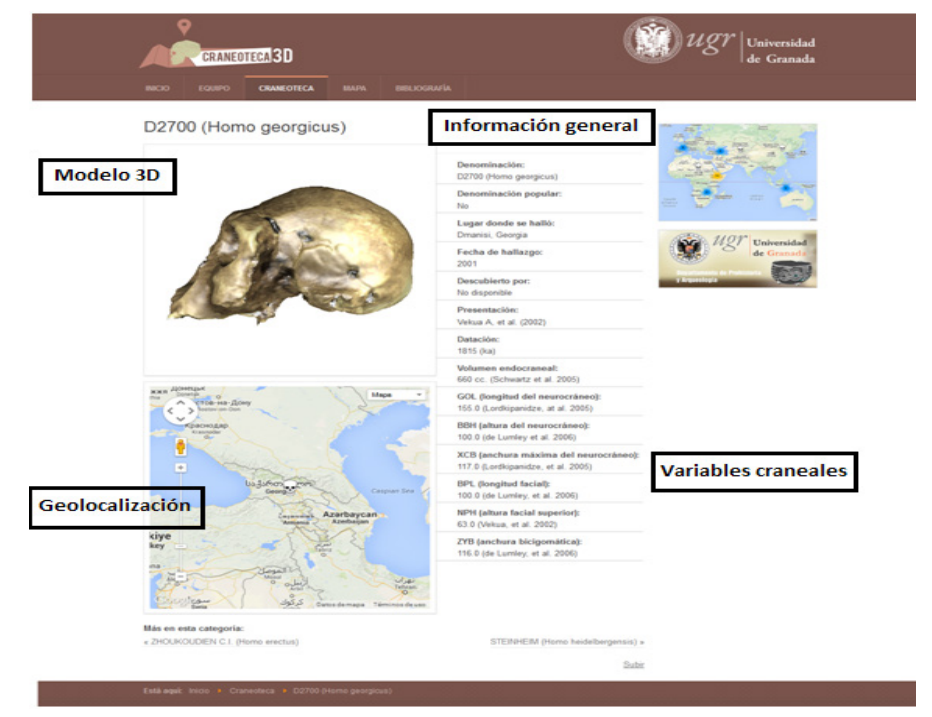

Fig. 3. Modelo de ficha (D2700) como aparece en la página web. 
Dicha información, junto con las reproducciones digitales tridimenisonales, configuran una base de datos alojada en una página web "la craneoteca del Dpto. de Prehistoria y Arqueología de la UGR", de libre acceso desde cualquier computadora con Internet desde la página del departamento (www.prehistoriayarqueologia.es/craneoteca).

En la página web se recogen los objetivos del proyecto y el equipo que lo ha llevado a cabo. En la pestaña de "craneoteca" se muestran en mosaico entre 5 y 6 individuos por página (disponiendo por el momento de 4 páginas), con su nombre y adscripción taxonómica. Seleccionando cada individuo se accede a la ficha individual (figura 3) de cada uno, donde se recoge la información anteriormente descrita. Además de los mapas indivualizados en cada ficha, hemos dispuesto una pestaña desde donde se accede e un mapamundi con la distribución de los yacimientos donde se han encontrado los fósiles de las réplicas digitalizadas. Los usuarios pueden interactuar con el mapa, por ejemplo, aumentado zoom en la zona elegida (efecto zoom) y enlazando desde los cráneos a la información sobre el cráneo en cuestión.

Tras un periodo de funcionamiento de la página hemos advertido algunos problemas. Para una correcta visualización de los modelos tridimensionales existen algunos requisitos, como tener un navegador web actualizado y una tarjeta gráfica reciente, con WebGL activado. Además, hay de que tener en cuenta que en la calidad del visionado de los modelos en 3D influyen las prestaciones del ordenador utilizado. Así, no en todos los ordenadores de las salas de informática de la Facultad de Filosofía y Letras de la Universidad de Granda ha sido posible visualizar correctamente la página, lo que limita nuestro objetivo "globalizador".

Se está considerando la posibilidad de crear las fichas en formato .PDF para repartir entre el alumnado, puesto que es un formato accesible y funcional (Niven et al., 2009). Las últimas versiones del Adobe Acrobat (Reader 7.0 o superior y Profesional 8.0 o superior) incorporan la posibilidad de gestionar modelos tridimensionales, que permiten ser visualizados, ampliados y rotados, configurar diversos parámetros de la visualización (como la iluminación o el fondo) e incluso realizar proyecciones, mediciones y secciones. Se ha planteado realizar un archivo por cada individuo, incluyendo la información recolectada de cada uno, así como el modelo tridimensional, para evitar sobrecargar un único archivo, lo que dificultaría su uso. De esta manera, el alumnado dispondría de acceso a la craneoteca sin necesidad de internet, resultando un material docente extra muy atractivo y completo para el alumnado, quien dispondría de la libertad de relacionarse con la información a su elección.

Asimismo, contemplamos la posibilidad de implementar la colección mediante la digitalización de nuevos individuos, que puedan ser tanto nuevas adquisiciones del Departamento como fruto de intercambios colaborativos con otros centros. Los investigadores y los centros deberíamos imbuirnos de los conceptos 
de libre acceso a la información y del compartir, aspectos muy ligados al desarrollo de internet, puesto que esto solo puede repercutir positivamente en la investigación y el conocimiento. Sin duda, los avances de esta era digital, en concreto las tecnologías 3D, permitirían la conservación de las colecciones óseas arqueológicas y la creación de bases de datos enormes para compartir. En este sentido ya se están dando los primeros pasos: se está trabajando en la creación de una gran "librería osteológica virtual" de esqueletos prehistóricos del continente americano (Kuzminsky y Gardiner, 2012) y que cuenta con disponer los modelos tridimensionales al resto de los investigadores.

\section{Conclusiones}

Lo fragmentario, disperso y delicado que resultan los restos óseos que nos informan sobre el proceso de hominización hace de este material algo único y restringido. Las colecciones de réplicas de estos cráneos es algo común en museos, universidades y otras instituciones y resultan una herramienta clave para su estudio y divulgación.

Las tecnologías 3D nos han permitido la posibilidad de generar una colección de referencia virtual, dándole una mayor visibilidad y uso. Si bien está claro que los modelos digitales no pueden sustituir el material original, sí están resultado muy beneficiosas en el proceso de enseñanza-aprendizaje, en particular en el apartado dedicado al aprendizaje independiente, lo que incluye la formación y el inicio de la investigación. La posibilidad de interactuar con los modelos tridimensionales y disponer de la información de forma libre puede está mejorando la capacidad de aprendizaje y favorece el interés por el estudio de la evolución humana. Además la disposición de la colección en internet está incrementado la capacidad divulgativa de la misma, pudiendo el público general acceder a su uso.

Por tanto, la conjunción entre las tecnologías digitales y el mundo virtual-Internet, por un lado y la Arqueología y la Antropología (en sus vertientes investigadora, didáctica y divulgativa), por otro, contribuye de manera significativa a la democratización del conocimiento en un mundo hiperconectado.

\section{Bibliografía}

ALLAM, A.H., THOMPSON, R.C., WANN, L.S., MIYAMOTO, M.I., EL-HALIM NUR EL-DIN, A., ELI-MAKSOUND, G.A., AL-TOHAMY SOLIMAN, M., BADR, I., EL-RAHMAN AMER, H.A., SUTHERLAND, M.L., SUTHERLAND, J.D., THOMAS, G.S.: "Atherosclerosis in ancient Egyptian mummies: the Horus study". Journal of the American College of Cardiology 4, 2011, pp. 315-327. 
BANNING, E.B.: The Archaeologist's Laboratoy: the Analysis of Archaeological Data. Plenum Publishers, New York: 2000.

BARCELÒ, A., FORTE, M., SANDERS, D.H. (Eds.): Virtual reality in archaeology, Archeopress BAR International Series 843, Oxford: 2000.

BAYLAC, M., FRIESS, M.: "Fourier descriptors, Procrustes superimposition, and data dimensionality: an example of cranial shape analysis in modern human populations". In: SLICE, D.E. (Ed.), Modern Morphometrics in Physical Anthropology. Kluwer Academic/Plenum Publishers, New York, 2005, pp. 145-165

BETTS, M.W., MASCHNER, H.D.G., SCHOU, C.D., SCHLADER, R., HOLMES, J., CLEMENT, N., SMUIN, M.: "Virtual zooarchaeology: building a web-based reference collection of northern vertebrates for archaeofaunal research and education". Journal of Archaeological Science 38, 2011, pp. 755-762.

BÖHLER, W., BORDAS VICENT, M., HEINZ, M., MARBS, A. y MÜLLER, H.: "High Quality Scanning and Modeling of Monuments and Artifacts". WSA2 Modelling and Visualization, WSA2.2 High Quality Scanning and Modeling of Monuments and Artifacts, Athens, Greece, May 22-27, 2004, pp. 1-17.

BRETZKE, K.\& CONARD, N. J.: "Evaluating morphological variability in lithic assemblages using 3D models of stone artefacts". Journal of Archaeological Science, 39(12), 2012, pp. 3741-3749.

BRUNO, F., BRUNO, S., DE SENSI, G., LUCHI, M.M., MANCUSO, S., MUZZUPAPPA, M.: "From 3D reconstruction to virtual reality: A complete methodology for digital archaeological exhibition". Journal of Cultural Heritage 11, 2010, pp. 42-49.

CONLOGUE, G., BECKETT, R., BAILEY, Y., POSH, J., HENDERSON, D., DOUBLE, G., KING, T.: "Paleoimaging: the use of radiography, magnetic resonance, and endoscopy to examine mummified remains". Journal of Radiology Nursing 27, 2008, pp. 5-13.

DAWSON, P.C., LEVY, R.M.: "A three-dimensional model of a Thule Inuit whale bone house". Journal of Fields Archaeology, 30, 2005, pp. 443-455.

DONEUS, M., NEUBAUER y W. STUDNICKA, N.: "Digital Recording of Stratigraphic Excavations", Proceedings of XIXth International Symposium CIPA 2003 New perspectives to the save cultural heritage, The CIPA International Archives for Documentation of Cultural Heritages 19, 2003, pp. 451-456.

FACCIA, K.J., WILLIAMS, R.C.: "Schmorl's nodes: clinical significance and implications for the bioarchaeological record". International Journal of Osteoarchaeology 18, 2008, pp. 28-44.

FRIESS, M.: "Calvarial shape variation among Middle Pleistocene hominins: an application of surface scanning in palaeoanthropology". Comptes Rendus Palevol 9, 2010, pp. 435-443. 
GROSMAN, L., SMIKT, O., \& SMILANSKY, U.: "On the application of 3-D scanning technology for the documentation and typology of lithic artifacts". Journal of Archaeological Science, 35(12), 2008, pp. 3101-3110.

GUNZ, P., MITTEROECKER, P., NEUBAUER, S., WEBER, G.W., BOOKSTEIN, F.L.: "Principles for the virtual reconstruction of hominin crania". Journal of Human Evolution, 57 (1), 2009, pp. 48-62

HENNESSY, R.J., STRINGER, C.B.: "Geometric morphometric study of the regional variation of modern human craniofacial form". American Journal of Physical Anthropology 117, 2002, pp. 37-48.

HINZEN, K.G., SCHREIBER, S., ROSELLEN, S.: "A high resolution laser scanning model of the Roman theatre in Pinara, Turkey - comparison to previous measurements and search for the causes of damage". Journal of Cultural Heritage, 14, 2013, pp. 424-430.

KAMPEL, M., MARA, H., SABLATNIG, R.: "Robust 3D reconstruction of archaeological pottery based on concentric circular rills". In: Magnenat- Thalmann, N., Rindel, J.H. (Eds.), Proceedings of the Sixth International Workshop on Image Analysis for Multimedia Interactive Services (WIAMIS'05), Montreux, Switzerland, 2005, pp. 14-20.

KAPPELMA, J., RYAN, T., ZYLSTRA, M., ALPORT, L., DEOLIVERIRA, M., GORDON, A., SCOTT, R.: "eSkeletons: a web-based platform for learning anatomical form and function". American Journal of Physical Anthropology 111, 192, 2000. Supplement. 30:192

KAPPELMA, J., MAGA, M., RYAN, T., ZYLSTRA, M., ALPORT, L., FESEHA, M.: "A web site for the study of human and primate comparative anatomy". American Journal of Physical Anthropology 114, 88, 2001. www.e-Skeletons.org.

KUZMINSKY, S.C., GARDINER, M.S.: "3D-dimensional laser scanning potential uses for museum conservation and scientific research". Journal Of Archaeological Science 39, 2012, pp. 2744-2751.

LEPOURAS, G., VASSILAKIS, C.: "Virtual museums for all: employing game technology for edutainment". Virtual reality 8, 2005, pp. 96-106.

LORDKIPANIDZE, D., PONCE DE LEÓN, M., MARGVELASHVILI, A., RAK, Y., RIGHTMIRE, P., VEKUA, A., ZOLLIKOFER, C.P.E.: "A Complete Skull from Dmanisi, Georgia, and the Evolutionary Biology of Early Homo". Science 10/2013; 342(6156):326-31

LYONS, P.D., RIOUX, M., PATTERSON, R.T.: "Application of a three-dimensional colour laser scanner to paleontology: an interactive model of a juvenile Tylosaurus sp. basisphenoid-basioccipital". Palaeontologia Electronica 3, 2000, a4.

MARTÍN LERONES, P., LLAMAS FERNÁNDEZ, J., MELERO GIL, A., GÓMEZ GARCÍA BERMEJO, J., ZALAMA CASANOVA, E.: "A practical approach to 
making accurate 3D layouts of interesting cultural heritage sites through digital models". Journal of Cultural Heritage 11, 2009, pp. 1-9.

MCPHERRON, S.P., GERNAT, T., HUBLIN, J.J.: "Structured light scanning for high-resolution documentation of in situ archaeological finds". Journal of Archaeological Science, 36, 2009, pp. 19-24.

MORALES, J.I., LORENZO, C. \& VERGÈS, J.M.: "Measuring Retouch Intensity in Lithic Tools: A new proposal using 3D scan data". Journal of Archaeological Method and Theory. 2013, DOI 10.1007/s10816-013-9189-0.

MOTANI, R.: "Detailed tooth morphology in a durophagus ichthyosaur captured by 3D laser scanner". Journal of Vertebrate Paleontology 25, 2005, pp. 462465.

MOSTAZA PÉREZ, T.; ZANCAJO JIMENO, J.J; LÓPEZ QUIROGA,J.; MARTÍNEZ TEJERA, A.: "Aplicación del escáner láser 3D a la documentación espacial de yacimientos arqueológicos". Actas del VII Congreso Ibérico de Arqueometría, Teruel, 2010, 403-408.

NIVEN, L., STEELE, T.E., FINKE, H., GERNAT, T., HUBLIN, J.J.: “Virtual skeletons: using a structured light scanner to create a 3D faunal comparative collection". Journal of Archaeological Science 36, 2009, pp. 2018-2023.

ÖHRSTRÖM, L., BITZER, A., WALTHER, M., RÜHLI, F.J.: "Technical note: terahertzimaging of ancient mummies and bone". American Journal of Physical Anthropology 142, 2010, pp. 497-500.

PANAGIOTOPOULOU, O.: "Finite element analysis (FEA): applying an engineering method to functional morphology in anthropology and human biology". Annals of Human Biology 36, 2009, pp. 609-623.

PFISTERER, T., BOOKSTEIN, F.L., BREUCKMANN, B., SCHAEFER, K., VIOLA, T.B., WOERNER, H., SEIDLER, H.: "The variability of the proximal femur in catarrhines - a new 3D method for describing anatomical structures". American Journal of Physical Anthropology 132 (S44), 2007, pp. 188-189.

PLYUSNIN, I., EVAN, A.R., KARME, A., GIONIS, A., JERNVALLI, J.: "Automated 3D phenotype analysis using data mining". PLoS One 3, 2008, e1742.

RELVAS, C., RAMOS, A., COMPLETO, A., SIMÔES, J.A., 2011. "The influence of data shape acquisition process and geometric accuracy of the mandible for numerical simulation". Computer Methods in Biomechanics and Biomedical Engineering 14, 2011, pp. 721-728.

SAITOU, N., KIMURA, R., FUKASE, H., YOGI, A., MURAYAMA, S., ISHIDA, H.: "Advanced CT images reveal nonmetric cranial variations in living humans". Anthropological Science 119, 2011, pp. 231-237.

SHOLTS, S.B., WALKER, P.L., KUZMINSKY, S.C., MILLER, K.W.P., WÄRMLÄNDER, S.K.T.S.: "Identification of group affinity from cross-sectional con- 
tours of the human midfacial skeleton using digital morphometrics and 3D laser scanning technology". Journal of Forensic Sciences 56, 2011, pp. 333-338.

SHOTT, M. J., \& TRAIL, B. W.: "Exploring new approaches to lithic analysis: Laser scanning and geometric morphometrics". Lithic Technology, 35(2), 2010, pp. 195-220.

SMITH, N.E., STRAIT, S.G.: "PaleoView3D: from specimen to online digital model. Paleontologia Electronica 11, 2008, 11A.

STÉPHANIE, A., CRETTÉ, S.A., NÄSÄNEN, L.M., GONZÁLEZ-PEREYRA, N.G., RENNISON, B.: "Conservation of waterlogged archaeological corks using supercritical CO2 and treatment monitoring using structured-light 3D scanning". Journal of Supercritical Fluids, 79, 2013, pp. 299-313

STRAIT, S.G., SMITH, N.E.: "PaleoView3D: an interactive database of mammals from the Paleocene/Eocene boundary. Journal of Vertebrate Paleontology 26, 2006, 129A

TOCHERI, M.W.: "Laser scanning: 3D analysis of biological surfaces". In: SENSEN, C.W., HALLGRIMSSON, B. (Eds.), Advanced Imaging in Biology and Medicine. Springer-Verlag, Berlin, 2009, pp. 85-101

WEBER, G.W., BOOKSTEIN, F.L.: Virtual Anthropology: a Guide to a New Interdisciplinary Field. Springer Wein, New York, 2011.

ZOLLIKOFER, C P; PONCE DE LEON, M.S.: "Early Homo from Dmanisi and its relationship to African and Asian Homo erectus". In: INDRIATI, E. Recent Advances on Southeast Asian Paleoanthropology and Archaeology. Jakarta, 2008, pp. 61-69.

ZOLLIKOFER, P.E., PONCE DE LEÓN, M.S., LIBERMA, D.E., GUY, F., PILBEAM, D., LIKIUS, A., MACKAYE, H.T., VIGNAUD, P., BRUNET, M.: "Virtual cranial reconstruction of Sahelanthropus tchadensis". Nature, 2005; 434(7034), pp. 755-9

ZOLLIKOFER, P.E., PONCE DE LEÓN, M.S., VANDERMEERSCH, B., LEVEQUE, F.: "Evidence for interpersonal violence in the St. Cesaire Neanderthal". Proceedings of the National Academy of Sciences, 2002; 99(9), pp. 6444-8

ZOLLIKOFER, P.E., PONCE DE LEÓN, M.S.: "Visualizing patterns of craniofacial shape variation in Homo sapiens". Proceedings of the Royal Society B: Biological Sciences, 2002; 269(1493):801-7

ZOLLIKOFER, P.E., PONCE DE LEÓN, M.S., MARTIN, R.D.,STUCKI, P.: “Neanderthal computer skulls". Nature, 1995; 375(6529), pp. 283-5

http://www.eva.mpg.de/evolution última consulta 12/04/2016 http://humanorigins.si.edu/evidence/3d-collection última consulta 12/04/2016 
